\title{
RESEARCH
}

Open Access

\section{Placental chorionic plate-derived mesenchymal stem cells ameliorate severe acute pancreatitis by regulating macrophage polarization via secreting TSG-} 6

Qilin Huang ${ }^{1,2+}$, Xiumei Cheng ${ }^{3 \dagger}$, Chen Luo ${ }^{4}$, Shuxu Yang ${ }^{2}$, Shuai Li ${ }^{1}$, Bing Wang ${ }^{1}$, Xiaohui Yuan ${ }^{1}$, Yi Yang ${ }^{1}$, Yi Wen ${ }^{1}$, Ruohong Liu', Lijun Tang ${ }^{1 *}$ and Hongyu Sun ${ }^{1,5^{*}}$

\begin{abstract}
Background: Mesenchymal stem cells (MSCs) hold promising potential to treat systemic inflammatory diseases including severe acute pancreatitis (SAP). In our previous study, placental chorionic plate-derived MSCs (CP-MSCS) were found to possess superior immunoregulatory capability. However, the therapeutic efficacy of CP-MSCs on SAP and their underlying mechanism remain unclear.

Methods: The survival and colonization of exogenous CP-MSCs were observed by bioluminescence imaging and CM-Dil labeling in rodent animal models of SAP. The therapeutic efficacy of CP-MSCs on SAP rats was evaluated by pathology scores, the levels of pancreatitis biomarkers as well as the levels of inflammatory factors in the pancreas and serum. The potential protective mechanism of CP-MSCs in SAP rats was explored by selectively depleting M1 or M2 phenotype macrophages and knocking down the expression of TSG-6.

Results: Exogenous CP-MSCs could survive and colonize in the injured tissue of SAP such as the lung, pancreas, intestine, and liver. Meanwhile, we found that CP-MSCs alleviated pancreatic injury and systemic inflammation by inducing macrophages to polarize from M1 to M2 in SAP rats. Furthermore, our data suggested that CP-MSCS induced M2 polarization of macrophages by secreting TSG-6, and TSG-6 played a vital role in alleviating pancreatic injury and systemic inflammation in SAP rats. Notably, we found that a high inflammation environment could stimulate CP-MSCs to secrete TSG-6.
\end{abstract}

\footnotetext{
*Correspondence: tanglj2016@163.com; shongyu2008@163.com

${ }^{\dagger}$ Qilin Huang and Xiumei Cheng contributed equally to this work.

'Department of General Surgery \& Pancreatic Injury and Repair Key

Laboratory of Sichuan Province, The General Hospital of Western Theater Command, Chengdu 610083, China

Full list of author information is available at the end of the article
}

C C The Author(s). 2021 Open Access This article is licensed under a Creative Commons Attribution 4.0 International License, which permits use, sharing, adaptation, distribution and reproduction in any medium or format, as long as you give appropriate credit to the original author(s) and the source, provide a link to the Creative Commons licence, and indicate if changes were made. The images or other third party material in this article are included in the article's Creative Commons licence, unless indicated otherwise in a credit line to the material. If material is not included in the article's Creative Commons licence and your intended use is not permitted by statutory regulation or exceeds the permitted use, you will need to obtain permission directly from the copyright holder. To view a copy of this licence, visit http://creativecommons.org/licenses/by/4.0/. The Creative Commons Public Domain Dedication waiver (http://creativecommons.org/publicdomain/zero/1.0/) applies to the data made available in this article, unless otherwise stated in a credit line to the data. 
Conclusion: Exogenous CP-MSCS tended to colonize in the injured tissue and reduced pancreatic injury and

systemic inflammation in SAP rats through inducing M2 polarization of macrophages by secreting TSG-6. Our study

provides a new treatment strategy for SAP and initially explains the potential protective mechanism of CP-MSCs on

SAP rats.

Keywords: Mesenchymal stem cells, Placenta, Severe acute pancreatitis, Macrophage polarization, TSG-6

\section{Introduction}

Severe acute pancreatitis (SAP) is a deadly inflammatory disease caused by local pancreatic lesions, and excessive hyperinflammation caused by immune imbalance is an important cause of systemic inflammatory response syndrome (SIRS) and secondary organ dysfunction $[1,2]$. Despite intensive care treatment of SAP has improved significantly during the past few decades, the therapeutic efficacy of SAP remains unsatisfactory, with severe complications and a high mortality rate [3]. Until now, the clinical treatment strategy of SAP is still mainly based on symptomatic supportive treatment and anti-inflammatory treatment, but these treatment strategies cannot effectively correct immune imbalance that leads to excessive hyperinflammation. Therefore, it is urgent to seek for a new therapeutic strategy to re-shape the body's immune balance in SAP.

Mesenchymal stem cells (MSCs) retain promising potential in the treatment of various inflammatory and immune diseases due to their remarkable anti-inflammatory and immunoregulatory capabilities [4-7]. However, numerous studies have demonstrated that MSCs derived from different tissues have some unique biological characteristics [8-11]. Most thrilling of all, some studies have confirmed that placentalderived MSCs (P-MSCs) not only have the advantages of rich tissue sources, easy noninvasive access, and few ethical restrictions, but also possess stronger immunoregulation and proliferation capacity. For instance, Talwadekar et al. found that P-MSCs were superior in terms of their expansion ability and immunoregulatory properties to that of umbilical cord-derived MSCs (UC-MSCs) [12]. In our prior research, we isolated and expanded three kinds of P-MSCs from different parts of the placenta, including chorionic plate-derived MSCs (CP-MSCs), chorionic villi-derived MSCs (CV-MSCs), and decidua-derived MSCs (D-MSCs), and found that CPMSCs had stronger proliferation and migration ability than other P-MSCs and UC-MSCs [13]. Excitingly, we found that CP-MSCs expressed CD106 higher than the other three MSCs and showed stronger ability in regulating macrophage polarization from M1 to M2 [13]. Consistent with this, a study showed that $\mathrm{CD}_{106^{+}}$MSCs possessed stronger proliferation and immunoregulation capabilities than $\mathrm{CD}_{106}{ }^{-}$ MSCs [14]. Considering that a superior source of MSCs is crucial for cell therapy, we thus chose CP-MSCs for the treatment of SAP.

During SAP, immune imbalance triggers inflammatory cascades that lead to SIRS, multiple organ dysfunction, and even death. As a critical participator in the immune system, macrophages play a vital role in the occurrence, development, and evolution of SAP [15-17]. Intriguingly, macrophages possess strong plasticity and change their functional phenotype dependent on the local microenvironment. Some studies have confirmed that the transformation of macrophages from M1 phenotype to M2 phenotype could reduce tissue damage in various inflammatory diseases [18-20]. For instance, human bone marrow-derived MSCs (BM-MSCs) alleviate lung injury by inducing M2 polarization of macrophages in acute respiratory distress syndrome [21]. Furthermore, our group previously demonstrated that regulating the M2 polarization of peritoneal macrophage through abdominal paracentesis drainage could ameliorate systemic inflammation and pancreatic injury in SAP rats [22]. Therefore, inducing the M2 polarization of macrophages might help prevent the progression of SAP. In the present study, we transplanted exogenous CP-MSCs into rodent animal models of SAP and systematically evaluated the protective effects of CP-MSCs on SAP rats; meanwhile, we explored the regulation and potential mechanism of CP-MSCs on macrophage polarization.

\section{Materials and methods \\ Establishment of the SAP model}

Healthy wild-type male Sprague Dawley (SD) rats weighing 200 220 g were purchased from Chengdu Dossy Experimental Animal Co., Ltd. (Chengdu, China) and fed in a suitable environment with $25^{\circ} \mathrm{C}$ and $12 \mathrm{~h}$ dark/light cycle, given free access to water and food. Experimental procedures were approved by the Institutional Animal Care and Use Committee at the General Hospital of Western Theater Command and carried out in accordance with the established International Guiding Principles for Animal Research. The rats were fasted for $12 \mathrm{~h}$ before all surgical procedures. All experimental animals were anesthetized with isoflurane (RWD Life Science, Shenzhen, China) during the operation. The SAP models were induced by retrograde injection of $4 \%$ sodium taurocholate (TCA, $1 \mathrm{ml} / \mathrm{kg}$ body weight, Sigma, USA) into the common biliopancreatic duct as previously described [23].

\section{Isolation, expansion, and identification of CP-MSCs}

$\mathrm{CP}-\mathrm{MSCs}$ derived from the human placental chorionic plate and were cultured in MSC serum-free media 
(Yocon, China). The specific experimental methods of CP-MSC isolation, expansion, and identification are detailed in our previous research [13]. Immunophenotypic analysis and osteogenic and adipogenic differentiation experiments confirmed that CP-MSCs isolated from human placental chorionic plate MSCs meet the criteria of MSCs proposed by the International Society for Cellular Therapies (Fig. S1).

\section{Bioluminescence imaging}

First, CP-MSCs were infected with lentivirus carrying a luciferase gene, and then CP-MSCs stably expressing luciferase were selected. Kunming mice were anesthetized, and $4 \%$ sodium taurocholate was injected through the pancreaticobiliary duct to prepare the SAP model. At 6 $\mathrm{h}$ after the operation, $1 \times 10^{6} \mathrm{CP}$-MSCs expressing luciferase were infused through the tail vein. Observe the survival and distribution of CP-MSCs in SAP mice at 1 h, 24 h, 72 h, 5 days, and 7 days after exogenous CPMSC transplantation. D-Luciferin $(150 \mathrm{mg} / \mathrm{kg}$ body weight) was administered i.p. to each mouse $10 \mathrm{~min}$ prior to imaging. Mice were then placed in an In Vivo Imaging System (IVIS) and the photons/second emitted from the tissues were quantified using Living Image software v3.2 (Caliper Life Sciences, Alameda, CA).

\section{CP-MSCs in vivo tracking}

Add $5 \mu$ g Cell Tracker ${ }^{\text {Tx }}$ CM-DiI Dye (Invitrogen, USA) to $4 \times 10^{6} \mathrm{CP}$-MSCs, incubate at $37^{\circ} \mathrm{C}$ for $5 \mathrm{~min}$, and then incubate at $4^{\circ} \mathrm{C}$ for $15 \mathrm{~min}$. After the incubation, wash twice with $1 \times$ PBS buffer to remove unbound CM-Dil Dye, then resuspend the cell pellet in PBS buffer, and adjust the cell concentration to $2 \times 10^{6}$ cells $/ \mathrm{ml}$. Ten SD rats were randomly divided into 2 groups: control group and SAP group (5 per group). CP-MSCs labeled with CM-Dil $\left(1 \times 10^{6}\right.$ cells $\left./ 100 \mathrm{~g}\right)$ were transplanted into rats via the tail vein at $6 \mathrm{~h}$ and $30 \mathrm{~h}$ after the operation. All rats were sacrificed $72 \mathrm{~h}$ after the first CP-MSC transplantation; the lung, heart, liver, pancreas, spleen, kidney, duodenum, and colon were collected and then fixed in $4 \%$ paraformaldehyde for $24 \mathrm{~h}$ and dehydrated in $30 \%$ sucrose solution. Subsequently, the tissues were embedded in Tissue Freezing Medium and cut into $8-\mu \mathrm{m}$-thick sections. The slides were washed with PBS and stained with DAPI to visualize the nuclei. The distribution of CP-MSCs in different organs was observed under a fluorescence microscope.

\section{CP-MSC transplantation in SAP rats}

Thirty-two SD rats were randomly divided into 4 groups: control group, control + CP-MSC group, SAP group, and SAP + CP-MSC group (8 per group). In the CPMSC intervention group, CP-MSCs $\left(1 \times 10^{6}\right.$ cells/100g) were delivered through tail vein $6 \mathrm{~h}$ and $30 \mathrm{~h}$ after the operation. All rats were sacrificed $72 \mathrm{~h}$ after the first CP-
MSC transplantation, serum, liver, and pancreas tissues were collected.

\section{Histopathology}

Pancreas samples were fixed in $10 \%$ buffered formaldehyde, embedded in paraffin, and sectioned. The 4- $\mu \mathrm{m}-$ thick deparaffinized sections were stained with $H \& E$ for routine histology. According to the scoring criteria reported by Schmidt et al. [24], the degree of pancreatic edema, acinar cell necrosis, hemorrhage, and inflammatory infiltrate were scored. Five different fields were randomly observed under the microscope each slide.

\section{Cell Counting Kit-8 (CCK-8) assay}

The CCK-8 assay was used to detect the effect of CMDil on the proliferation of CP-MSCs. The detailed operating steps were seen in supplementary materials.

\section{Enzyme-linked immunosorbent assay (ELISA)}

Inflammatory factors (IL-1 $\beta$, IL-6, TNF- $\alpha$, TGF- $\beta$, IL-4, and IL-10), amylase, and lipase in rat serum were detected by ELISA kits (Shanghai Jianglai Biotech, China). In addition, the human tumor necrosis factor- $\alpha$-induced gene/protein 6 (TSG-6) ELISA kit (Shanghai Jianglai Biotech, China) was used to detect the concentration of TSG-6 in the culture supernatant of CP-MSCs. Detailed operating steps were according to the manufacturer's instructions.

\section{Detection of myeloperoxidase (MPO) activity in pancreatic tissue}

Accurately weigh pancreatic tissue of the same quality, then grind it into a homogenate, and follow the manufacturer's instructions in the kit to detect MPO activity in the pancreatic tissue of each group.

\section{Real-time quantitative PCR (RT-qPCR)}

Total RNA was extracted using Trizol reagent (Invitrogen Inc., USA), according to the products' instructions. The RNA was quantified by measuring the absorbance at $260 \mathrm{~nm}$ and $280 \mathrm{~nm}$ using a spectrophotometer (NanoDrop Technologies, USA). RT-qPCR was performed with a CFX96 Real-Time PCR Detection System (Bio-Rad, USA) using one-step SYBR PrimeScript RT-PCR Kit (TaKaRa, Japan). The sequences of primers are listed in supplementary Table S1.

\section{Immunofluorescence staining}

Immunofluorescence staining is used to detect the polarizing phenotype of macrophages in pancreas and liver tissues, and the detailed method is described in supplementary materials. 
Preparation and polarization induction of bone marrowderived macrophages

Bone marrow (BM)-derived macrophages were isolated from SD rats by flushing the BM with DMEM (Hyclone, USA) as previously described [25, 26]. Bone marrow macrophage induction medium was used to induce differentiation of precursor cells into macrophages. After 7-10 days in culture, nonadherent cells were removed and adherent cells were ready for experiment. Macrophages were induced with an M1 or M2 polarization induction medium for $24 \mathrm{~h}$ and then collected for subsequent experiments.

Bone marrow macrophage induction medium: DMEM + 10\% FBS (Gibico, USA), 10 ng/ml M-CSF (Peprotech, USA).

Macrophage M1 polarization induction medium: DMEM + 10\% FBS, $100 \mathrm{ng} / \mathrm{ml}$ LPS (Sigma, USA), 50 $\mathrm{ng} / \mathrm{ml}$ IFN- $\gamma$ (Peprotech, USA).

Macrophage M2 polarization induction medium: DMEM + 10\% FBS, 10 ng/ml IL-10 (Peprotech, USA), 10 ng/ml IL-13 (Peprotech, USA).

\section{Selective depletion of M1 or M2 macrophages In vitro experiment}

Unpolarized-induced macrophages (M0), M1 polarizationinduced macrophages (M1), and M2 polarization-induced macrophages (M2) were inoculated into six-well plates at $1 \times$ $10^{6}$ cells/well. After attachment of macrophages, $\mathrm{GdCl} 3(100$ $\mu \mathrm{M}$, Sigma, USA) or mannosylated clodronate-encapsulated liposomes (MCLs, Encapsula Nano Sciences, USA) were added to the macrophage medium. The volume ratio of MCLs to the culture medium is 1:100. Macrophages were collected for apoptosis analysis after $48 \mathrm{~h}$ of intervention.

\section{In vivo experiment}

Thirty-six SD rats were randomly divided into 6 groups: $\mathrm{SAP}$ group, $\mathrm{SAP}+\mathrm{CP}-\mathrm{MSC}$ group, $\mathrm{SAP}+\mathrm{GdCl} 3$ group, $\mathrm{SAP}+\mathrm{GdCl} 3+\mathrm{CP}-\mathrm{MSC}$ group, SAP + MCLs group, and SAP + MCLs + CP-MSC group (6 per group). In the $\mathrm{GdCl} 3$ intervention group, the $\mathrm{GdCl} 3$ solution $(0.5 \%$, $20 \mathrm{mg} / \mathrm{kg}$ ) was infused via the tail vein immediately after the operation. In the MCL intervention group, $1 \mathrm{ml}$ of MCL solution was infused via the tail vein immediately after the operation. In the CP-MSC intervention group, CP-MSCs $\left(1 \times 10^{6}\right.$ cells/100g) were delivered through the tail vein $6 \mathrm{~h}$ and $30 \mathrm{~h}$ after the operation. All rats were sacrificed $72 \mathrm{~h}$ after the first CP-MSC transplantation, and serum, liver, and pancreas tissues were collected.

\section{Flow cytometric analysis of macrophage apoptosis}

Macrophage apoptosis was detected using Annexin VFITC Apoptosis Detection Kit (Beijing Solarbio Science \& Technology Co., Ltd., China), and the detailed operating steps are described in supplementary materials.

\section{TSG-6 shRNA transfection}

CP-MSCs were transfected with TSG-6-specific or nonspecific control short hairpin A (shRNA, Shanghai Genechem Co., Ltd., China) using transfection reagent in shRNA transfection media according to the manufacturer's protocol. Puromycin (Sigma, USA) was employed to select stable knockdown cells for at least three passages.

\section{CP-MSC intervened with SAP rat serum}

To simulate the microenvironment of CP-MSCs in SAP rats, CP-MSCs were cultured in MSC serum-free media containing $0 \%, 25 \%$, and $50 \%$ SAP rat serum. After $12 \mathrm{~h}$ of cultivation, CP-MSCs were collected for RT-qPCR assay.

\section{Macrophages and CP-MSC noncontact co-culture}

To explore the effects of different polarized phenotype macrophages on CP-MSCs, CP-MSCs and macrophages (M1 or M2 macrophages) were co-cultured at 2:5 through transwell chamber $(0.4-\mu \mathrm{m}$ pore size; Corning, USA), where CP-MSCs and macrophages were located in the lower and upper compartment of the chamber respectively. After $24 \mathrm{~h}$ of co-cultivation, CP-MSCs were collected for RT-qPCR assay.

To explore the mechanism of CP-MSCs regulating macrophage polarization, $\mathrm{M} 1$ macrophages were inoculated in six-well plates, and then CP-MSCs (TSG-6 shRNA) or CP-MSCs (scr shRNA) were inoculated in the upper layer of the transwell chamber $(0.4-\mu \mathrm{m}$ pore size; Corning, USA); the ratio of macrophages to CPMSCs is 5:1. After 24 h of co-cultivation, the polarization phenotype of macrophages was detected by flow cytometry and RT-qPCR.

\section{Flow cytometry analysis}

The polarized phenotype of macrophages was analyzed using the following antibodies: FITC-conjugated CD163 (Bio-Rad, USA), PE-conjugated CD86 (BD Biosciences, USA), and Alexa-Flour647-conjugated CD68 (Bio-Rad, USA). Nonspecific isotype-matched antibodies served as controls. The cells were analyzed using a flow cytometry instrument (BD CantoII, USA) and the data were analyzed using FlowJo V10.

\section{Statistical analysis}

Statistics as well as graphical representations were performed using GraphPad Prism ${ }^{\text {mx }} 7.0$ (GraphPad Software Inc., USA). All data are expressed as the means \pm SEM. Comparisons between two groups were performed using Student's t-test. Comparisons between more than two groups were analyzed by a one-way ANOVA test. Results were considered statistically significant when $\mathrm{P}<$ 0.05 . 


\section{Results}

Survival and distribution of exogenous CP-MSCs in rodent animal models of SAP

The survival of exogenous CP-MSCs in the hyperinflammatory environment of SAP is the basis for therapeutic effects. Therefore, the bioluminescence imaging was used to monitor the survival status of CP-MSCs in SAP mice. The results showed that the number of surviving
CP-MSCs decreased significantly when CP-MSCs were transplanted into SAP for $72 \mathrm{~h}$, while no fluorescent signal could be detected in vivo after CP-MSCs were transplanted for 7 days (Fig. 1A). As CP-MSC migration and recruitment are crucial to the success of CP-MSCmediated immune regulation, we determined whether exogenous CP-MSCs may respond to signals of cellular damage to the sites of injury after SAP. To track the

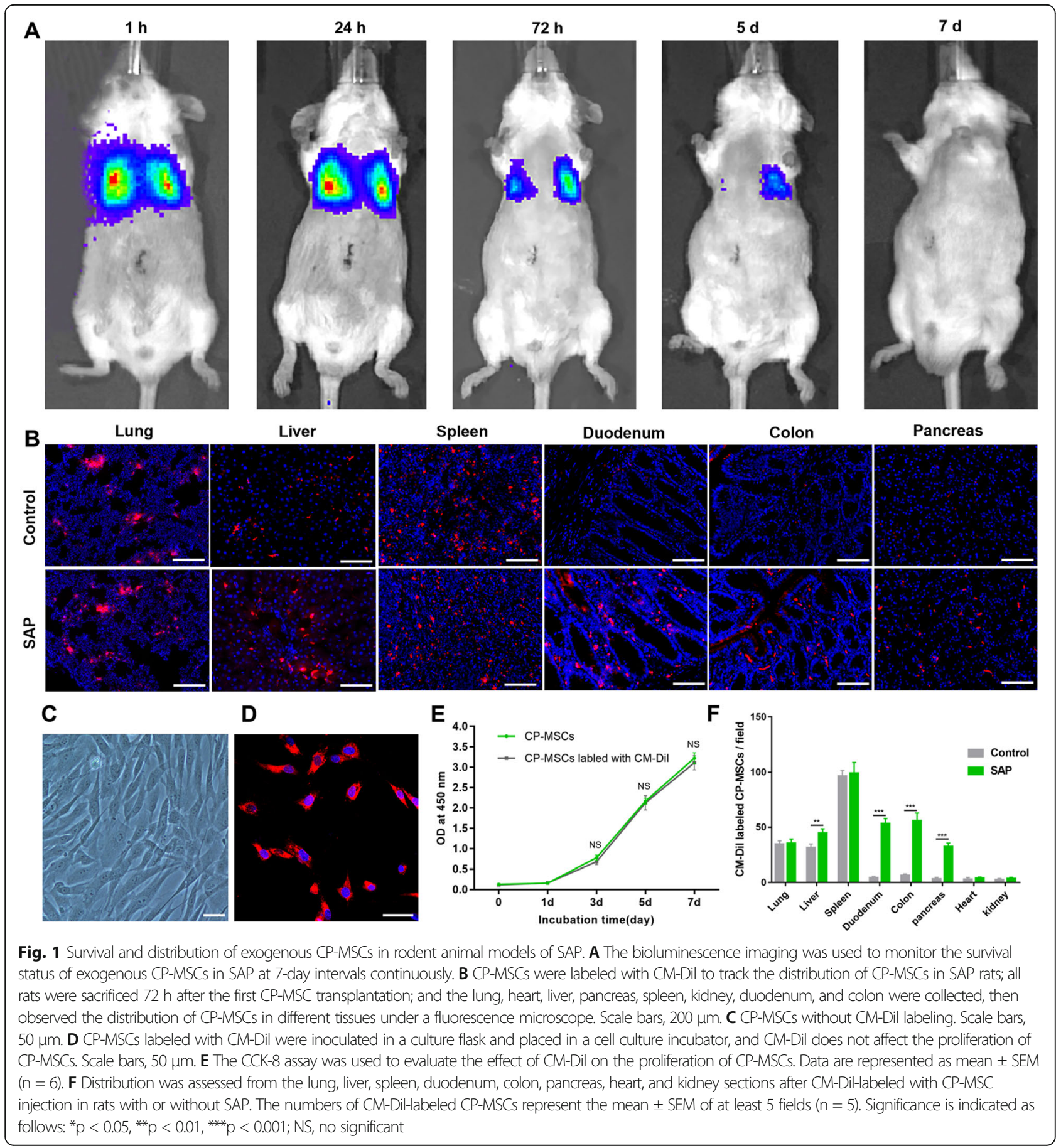


distribution and colonization of CP-MSCs in SAP rats, CP-MSCs were labeled with CM-Dil and adoptively transferred into SAP rats via tail vein. Indeed, we found that there were more CP-MSCs (red) colonized in the liver, pancreas, duodenum, and colon of the SAP group compared to the control group (Fig. 1B, F). Moreover, to observe whether CM-Dil affects the proliferation of CPMSCs, CP-MSCs were labeled with CM-Dil, inoculated in the culture flask, and placed in a cell culture incubator. We found that CM-Dil had no significant effects on the proliferation and morphology of CP-MSCs (Fig. 1C, D). Meantime, we confirmed that CM-Dil had no

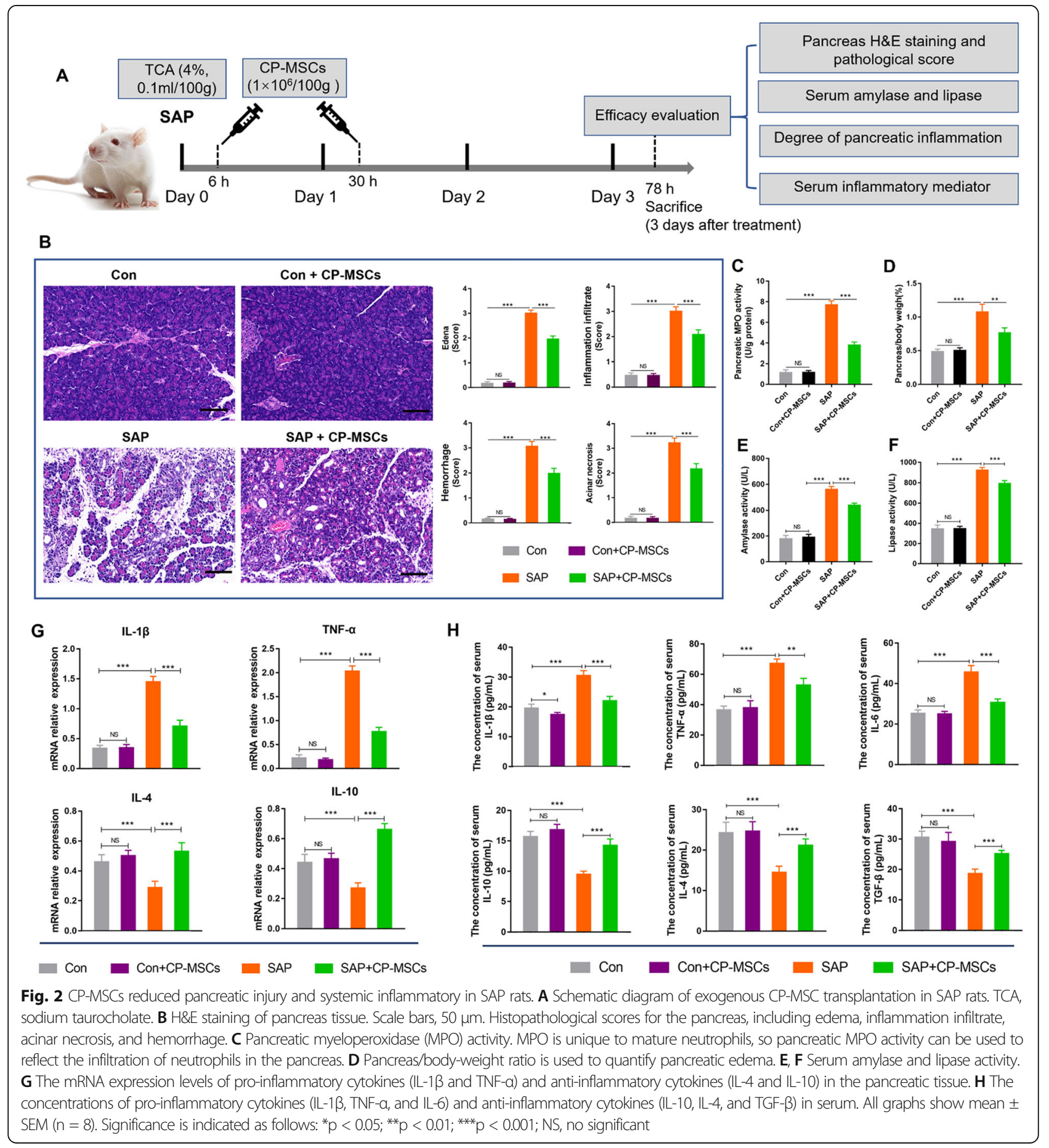


significant effects on the proliferation of CP-MSCs by CCK-8 assay (Fig. 1E).

\section{CP-MSCs could alleviate pancreatic injury and systemic inflammatory}

To assess the therapeutic efficacy of CP-MSCs on pancreatic injury in SAP rats, we first performed pancreatic histopathology scores and measured the activity of amylase and lipase in serum and pancreatic MPO activity (Fig. 2A). Histologically, the SAP group showed obvious morphological damage, such as edema, inflammation infiltrate, acinar necrosis, and hemorrhage, whereas the pancreatic tissue damage was significantly reduced in the SAP + CP-MSC group (Fig. 2B). In addition, compared with the SAP group, pancreatic MPO activity, pancreas/body-weight ratio, and serum amylase and lipase activity were significantly reduced in the SAP + CPMSC group (Fig. 2C-F). Meanwhile, RT-qPCR results of inflammatory factor mRNA in pancreatic tissue showed that CP-MSCs could significantly reduce the expression levels of pro-inflammatory factors IL- $1 \beta$ and TNF- $\alpha$ and increase the expression levels of anti-inflammatory factors IL-4 and IL-10 (Fig. 2G). Finally, we explored the effects of CP-MSCs on the systemic inflammatory in SAP

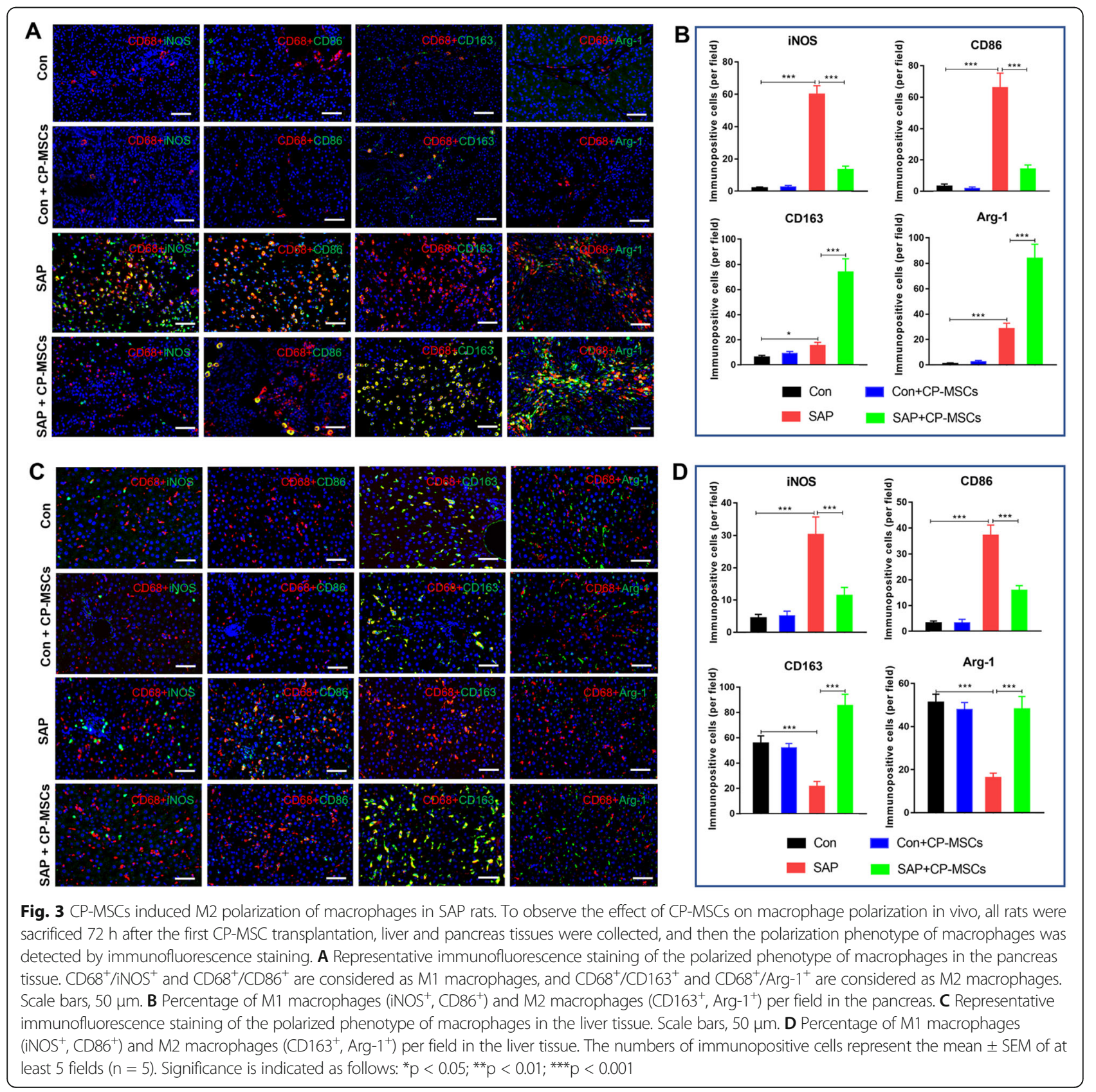




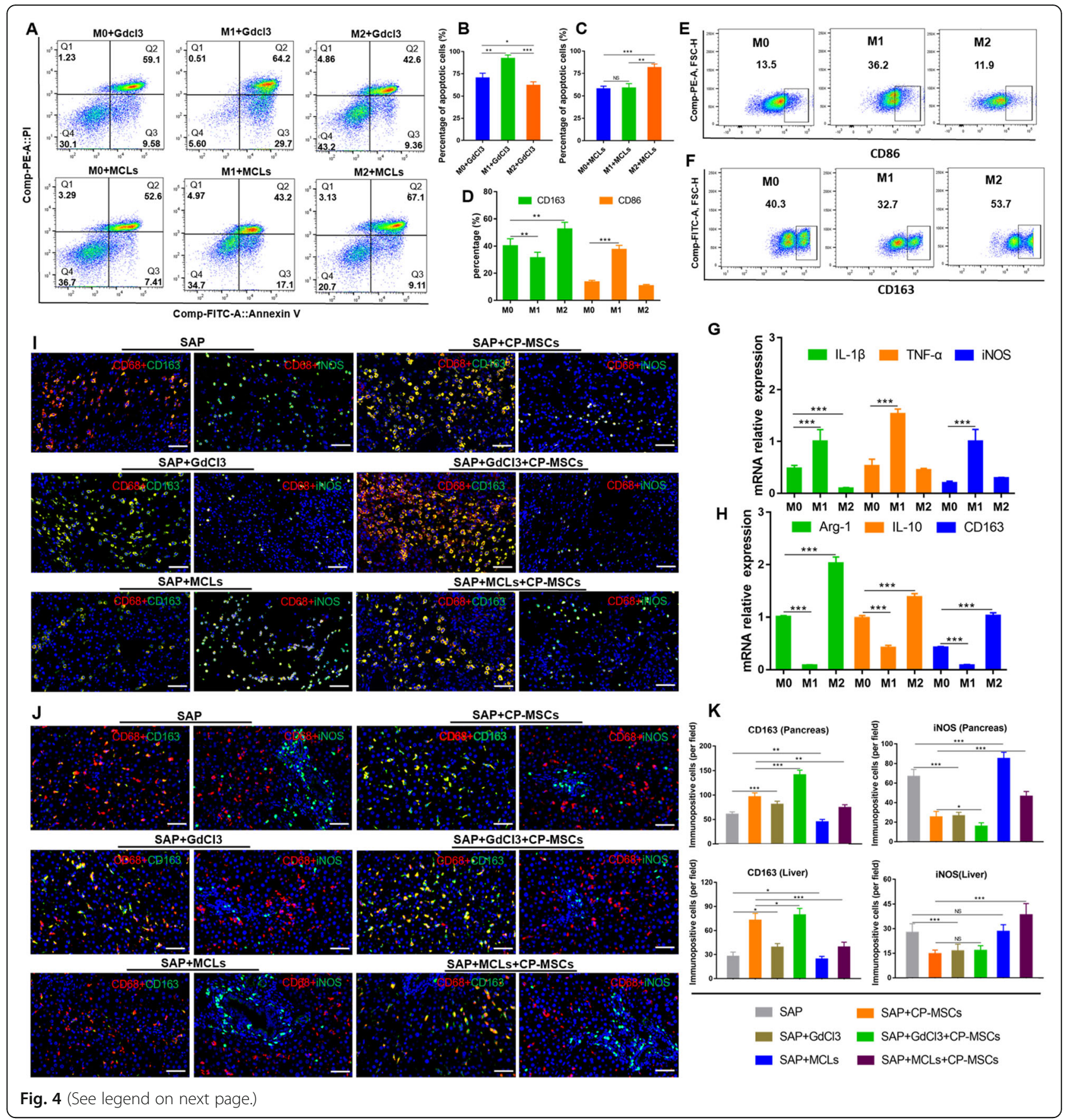




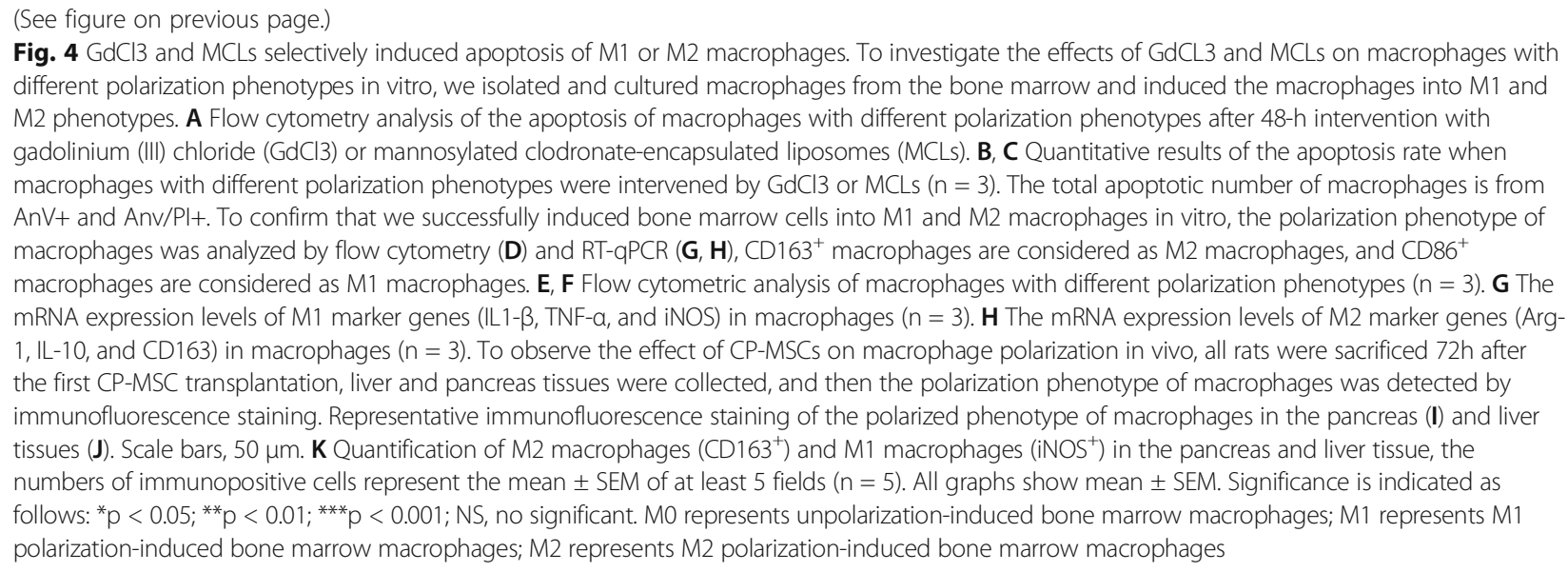

rats. ELISA experimental data showed that compared with the SAP group, in the SAP + CP-MSC group, the serum concentrations of pro-inflammatory cytokines (such as IL-1 $\beta$, TNF- $\alpha$, and IL-6) significantly decreased, while the concentrations of anti-inflammatory cytokines (such as IL-10, IL-4, and TGF- $\beta$ ) increased significantly (Fig. 2H). The above data fully illustrated that exogenous CP-MSCs could reduce pancreatic injury and systemic inflammation in SAP rats.

\section{CP-MSCs induced M2 polarization of macrophages}

Increasing evidence has shown that M2 polarization of macrophages could mitigate tissue inflammatory and damage $[21,27,28]$, so exploring the regulation of $\mathrm{CP}$ MSCs on macrophage polarization would provide strong evidence for revealing its potential therapeutic mechanism in SAP rats. The results of immunofluorescence staining of the polarized phenotype of macrophages showed that there were only a few macrophages $\left(\mathrm{CD}_{68}{ }^{+}\right)$in the pancreas tissues of the Con group and Con + CP-MSC group, and these macrophages were mainly located in the lobular space of the pancreas, beside blood vessels or bile ducts (Fig. 3A). Compared with the Con group, there were a large number of macrophages in the pancreas and liver tissues of the SAP group, and these macrophages highly expressed CD86 and iNOS, while very few expressed CD163 and Arg-1 (Fig. 3A-D). Compared with the SAP group, the number of macrophages in the pancreatic tissue of the SAP + CP-MSC group was slightly reduced, and the expression of CD86 and iNOS in the pancreas and liver tissue macrophages were significantly decreased, while the expression of CD163 and Arg-1 were significantly increased (Fig. 3A-D). Based on these results, we inferred that CP-MSCs could induce the polarization of macrophages from M1 to M2 in the pancreas and liver tissues of SAP rats.

\section{CP-MSCs mitigated pancreatic injury and systemic inflammatory mainly by inducing M2 polarization of macrophages}

To further explore whether CP-MSCs exerted a therapeutic role by regulating the polarization of macrophages from M1 to M2 in SAP rats, we selectively deplete M1 or M2 macrophages when CP-MSCs were administered, and then observe the protective effects of CP-MSC on SAP rats. We depleted M1 macrophages by administration of $\mathrm{GdCl} 3$, which upon phagocytosis induces apoptosis of inflammatory macrophages (M1) via competitive inhibition of $\mathrm{Ca}_{2}{ }^{+}$mobilization and damage to plasma membranes [29]. We depleted M2 macrophages using mannosylated clodronate liposomes (MCLs) that bind the mannose receptor which is upregulated following M2 polarization, and induce apoptosis via clodronate-mediated depletion of intracellular iron [30,31]. First, we successfully obtained macrophages from rat bone marrow (Figure S3) and induced them into $\mathrm{M} 1$ and $\mathrm{M} 2$ macrophages (Fig. 4D-H). Next, we confirmed that $\mathrm{GdCl} 3$ could relatively selectively induce apoptosis of M1 macrophages (Fig. 4A, B), and MCLs could relatively selectively induce apoptosis of M2 macrophages in vitro (Fig. 4A, C). Finally, $\mathrm{GdCl} 3$ or MCLs were infused into SAP rats through the tail vein before CP-MSC transplantation (Fig. 5A), and then the polarization phenotype of macrophages in the liver and pancreas was detected by immunofluorescence staining. We found that when SAP rats were given $\mathrm{GdCl} 3$ intervention, $\mathrm{iNOS}^{+}$macrophages in the pancreas and liver tissues decreased significantly, while $\mathrm{CD} 163^{+}$macrophages increased; when SAP rats were given MCL intervention, $\mathrm{CD}_{163^{+}}$macrophages in pancreas and liver tissues decreased significantly, while $\mathrm{iNOS}^{+}$macrophages increased (Fig. 4I-K). Therefore, the above data manifested that $\mathrm{GdCl} 3$ selectively depleted M1 macrophages, and MCLs selectively depleted M2 macrophages in the pancreas and liver tissues of SAP rats. 


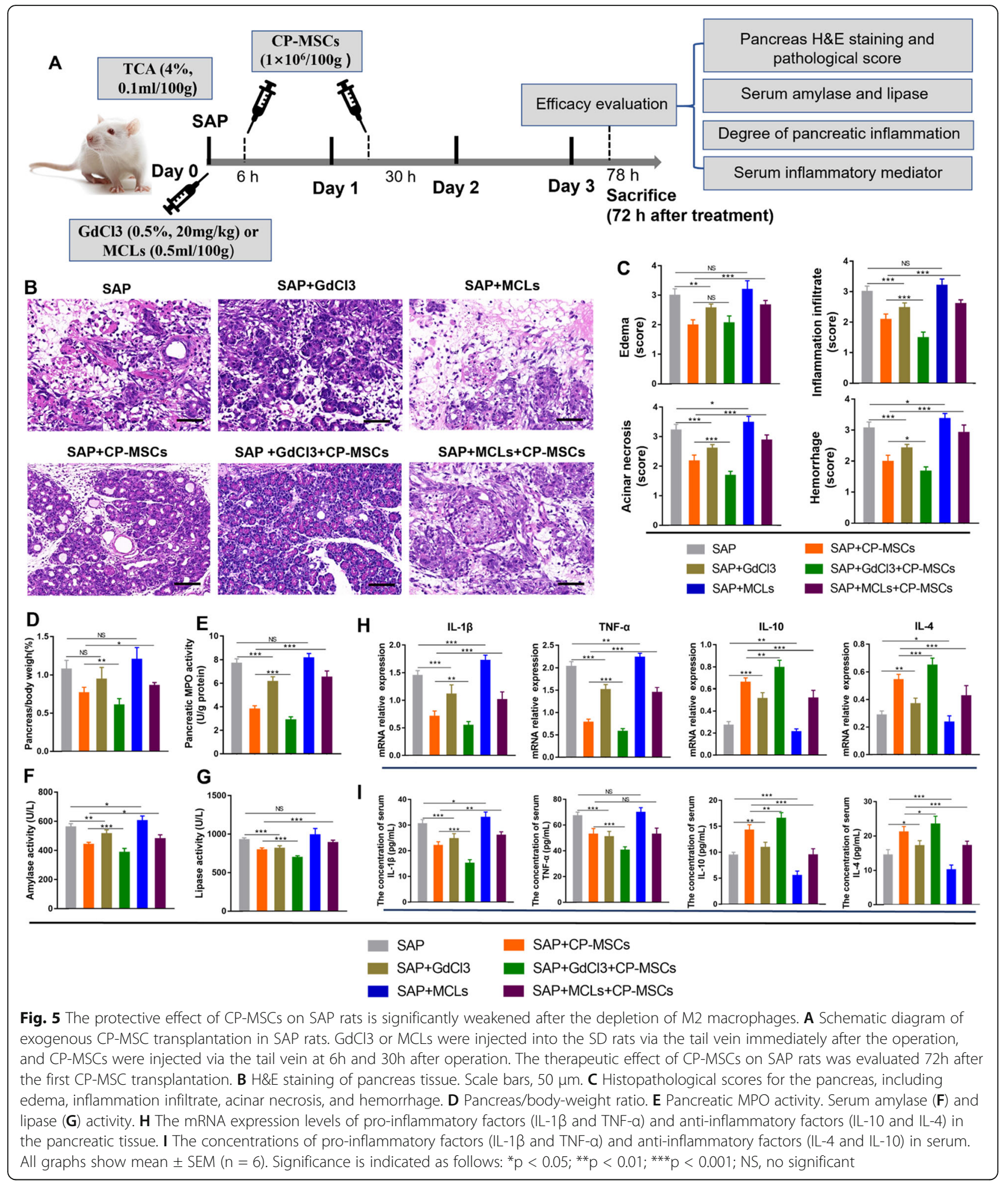

Moreover, through pancreatic $\mathrm{H} \& \mathrm{E}$ staining and pathological scores (Fig. 5B, C), the pancreas/bodyweight ratio (Fig. 5D), pancreatic MPO activity (Fig. 5E), RT-qPCR results of pancreatic inflammatory factors mRNA (Fig. 5H), and ELISA data of serum inflammatory factors (Fig. 5I), amylase (Fig. 5F), and lipase (Fig. 5G), we found that pancreatic damage and systemic inflammation were significantly reduced when M1 macrophages were depleted, and pancreatic damage and systemic inflammation were significantly worsened when 


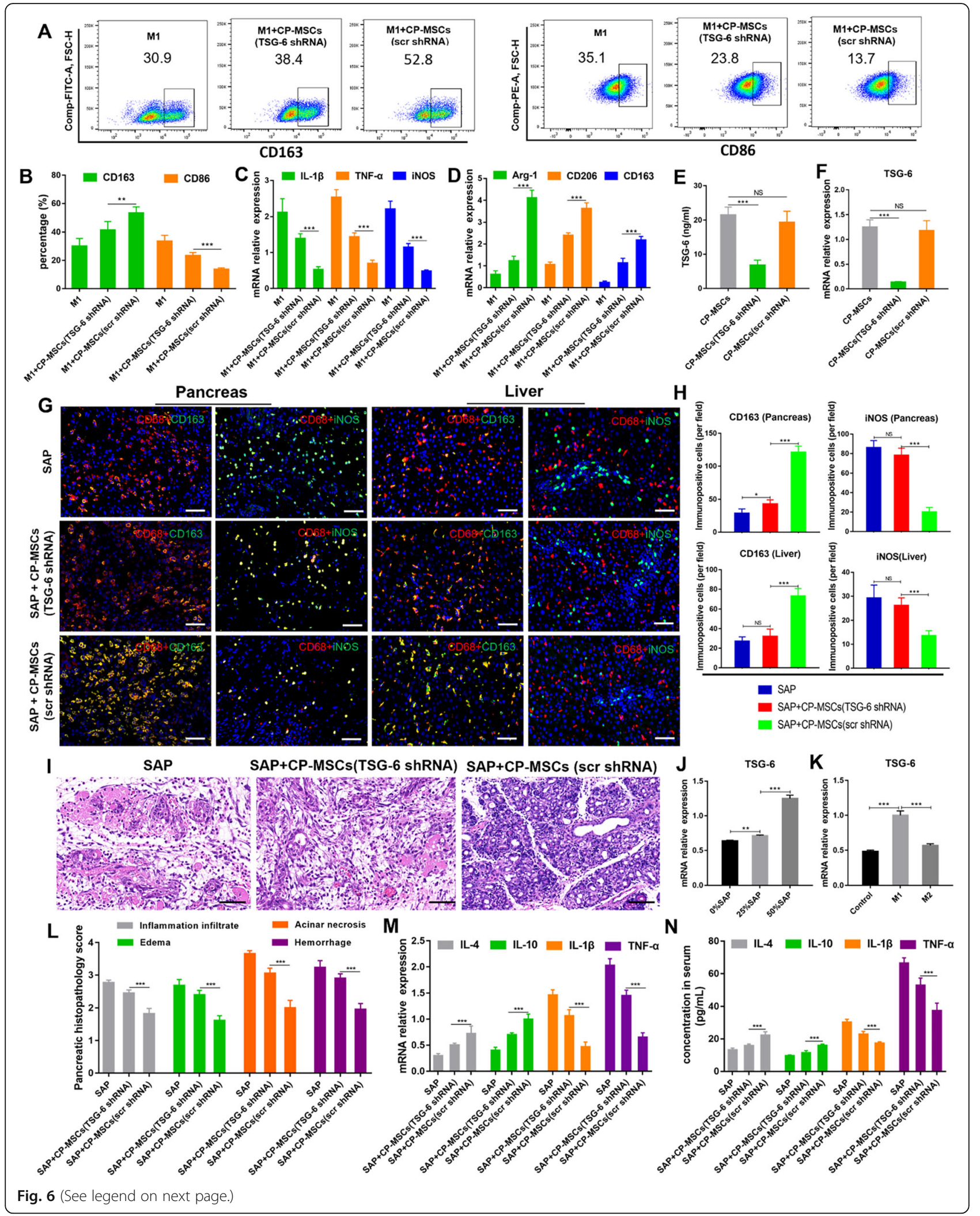


(See figure on previous page.)

Fig. 6 CP-MSC-derived TSG-6 played a vital role in inducing M2 polarization of macrophages and reducing pancreatic injury and systemic inflammation in SAP rats. A Flow cytometric analysis of the macrophage polarization phenotype after CP-MSCs (TSG-6 shRNA) or CP-MSCs (scr shRNA) co-cultured with M1 macrophages for $24 \mathrm{~h}$. B Quantification of macrophage polarization phenotype. CD86 ${ }^{+}$macrophages are considered as M1 macrophages, and $C D 163^{+}$macrophages are considered as M2 macrophages $(n=3)$. C The mRNA expression levels of M1 marker genes (IL1- $\beta$, TNF-a, and iNOS) in macrophages $(n=3)$. D The mRNA expression levels of M2 marker genes (Arg1, IL-10, and CD163) in macrophages ( $n$ = 3). TSG-6 protein (E) and mRNA (F) expression levels in CP-MSCs (TSG-6 shRNA), CP-MSCs (scr shRNA), and CP-MSCs ( $n=3$ ). G Representative immunofluorescence staining of the polarized phenotype of macrophages in the pancreas and liver tissue. Scale bars, $50 \mu \mathrm{m}$. $\mathbf{H}$ Quantification of $\mathrm{M} 2$ macrophages $\left(\mathrm{CD}_{163}{ }^{+}\right.$) and $\mathrm{M} 1$ macrophages (iNOS ${ }^{+}$) in the pancreas and liver tissue. The numbers of immunopositive cells represent the mean \pm SEM of at least 5 fields $(n=5)$. I H\&E staining of pancreas tissue. Scale bars, $50 \mu$ m. J RT-qPCR analysis of TSG-6 expression level of CPMSCs after intervention by different concentrations of SAP rat serum $(n=3)$. K RT-qPCR analysis of the TSG-6 expression level of CP-MSCs after CP-MSCs were co-cultured with M1 macrophages or M2 macrophages for $24 \mathrm{~h}(\mathrm{n}=3)$. L Histopathological scores for the pancreas showed the degree of edema, inflammation infiltrate, hemorrhage, and acinar necrosis $(n=5)$. $\mathbf{M}$ The mRNA expression levels of pro-inflammatory factors (IL$1 \beta$ and TNF-a) and anti-inflammatory factors (IL-4 and IL-10) in the pancreas $(n=5)$. $\mathbf{N}$ The concentrations of pro-inflammatory factors (IL-1 $\beta$ and TNF-a) and anti-inflammatory factors (IL-4 and IL-10) in rat serum $(n=5)$. Data are presented as mean \pm SEM. Significance is indicated as follows: ${ }^{*} \mathrm{p}<0.05 ;{ }^{* *} \mathrm{p}<0.01 ;{ }^{* * *} \mathrm{p}<0.001 ; \mathrm{NS}$, no significant

M2 macrophages were depleted. Therefore, it is showed that M1 macrophages aggravated tissue inflammation and injury in SAP rats, while M2 macrophages promoted the regression of tissue inflammation and repaired the injured tissues. In addition, we found that the therapeutic efficacy of CP-MSCs was significantly enhanced when M1 macrophages were depleted, while the therapeutic efficacy of CP-MSCs was significantly inhibited when M2 macrophages were depleted in SAP rats. Therefore, it is indicated that CP-MSCs attenuated pancreatic injury and systemic inflammation mainly by inducing M2 polarization of macrophages in SAP rats.

\section{CP-MSCs induced M2 polarization of macrophages by secreting TSG-6}

To verify that CP-MSCs regulate the polarization of macrophages from M1 to M2 by secreting TSG-6, RNA interference technology was used to inhibit the expression of TSG-6 in CP-MSCs. By measuring the concentration of TSG-6 in the culture supernatant (Fig. 6E) and the expression level of TSG-6 gene (Fig. 6F), it was confirmed that the expression of TSG-6 of CP-MSCs was successfully inhibited. Flow cytometric analysis showed that compared with the M1 + CP-MSC (scr shRNA) group, $\mathrm{CD} 163^{+}$macrophages were significantly reduced and $\mathrm{CD} 86^{+}$macrophages were significantly increased in the M1 + CP-MSC (TSG-6 shRNA) group (Fig. 6A, B). In addition, RT-qPCR results indicated that compared with the M1 + CP-MSC (scr shRNA) group, the mRNA expression levels of M1 macrophage marker genes (IL$1 \beta$, TNF- $\alpha$, and iNOS) were significantly increased, and M2 macrophage marker genes (Arg-1, IL-10, and CD163) were significantly reduced in the M1 + CP-MSC (TSG-6 shRNA) group (Fig. 6C, D). The above data manifested that TSG-6 secreted by CP-MSCs played an important role in regulating the polarization of macrophages from M1 to M2.

\section{CP-MSC-derived TSG-6 alleviated SAP by suppressing pancreatic and systemic inflammation}

In the preceding, we have shown that CP-MSC-derived TSG-6 shifted the macrophages from a proinflammatory phenotype (M1) to an anti-inflammatory phenotype (M2) in vitro. Next, we explored whether CPMSC-derived TSG-6 is involved in the switch of antiinflammatory macrophages in SAP rats. Polarized phenotype immunofluorescence staining of macrophages showed that when the expression of CP-MSC-derived TSG-6 was suppressed, iNOS ${ }^{+}$macrophages (M1) increased significantly and $\mathrm{CD} 163^{+}$macrophages (M2) decreased significantly in the pancreas and liver tissues of SAP rats (Fig. 6G, H).

To confirm that CP-MSCs exert a therapeutic role mainly by secreting TSG-6, CP-MSCs (TSG-6 shRNA) were transplanted into SAP rats to evaluate the therapeutic effect. Pancreatic HE staining and pathology scores (Fig. 6I, L), RT-qPCR results of inflammatory factors in pancreatic tissues (Fig. 6M), and ELISA data of serum inflammatory factors (Fig. 6N) indicated that when the expression of CP-MSC-derived TSG- 6 was suppressed, the therapeutic effect of CP-MSCs is significantly weakened. Therefore, it showed that TSG-6 secreted by CP-MSCs played a vital role in reducing pancreatic injury and systemic inflammation.

Moreover, we found that when SAP rat serum was used to simulate the hyperinflammatory environment on CP-MSCs in SAP rats, SAP rat serum significantly stimulated CP-MSCs to express TSG-6 in a dose-dependent manner (Fig. 6J). In addition, we found that when $\mathrm{CP}$ MSCs were co-cultured with macrophages, M1 macrophages stimulated CP-MSCs to express more TSG-6, while M2 macrophages did not significantly affect the expression of TSG-6 in CP-MSCs (Fig. 6K). Therefore, the above results indicated that a hyperinflammatory environment could stimulate CP-MSCs to express more TSG-6. 


\section{Discussion}

In the present study, we provided the first evidence that exogenous CP-MSCs attenuated SAP by inducing macrophage polarization from M1 to M2 via secreting TSG-6. The important findings of this study are as follows: (i) Exogenous CP-MSCs can survive in the hyperinflammatory environment of SAP and tend to colonize the injured tissue, such as the pancreas, lung, liver, and intestine; (ii) CP-MSCs alleviate pancreatic injury and systemic inflammatory by inducing macrophage polarization from M1 to M2 in SAP rats; (iii) CP-MSCs secrete more TSG-6 in the inflammatory environment of SAP, thereby inducing macrophages to polarize from M1 to M2; (iv) TSG-6 secreted by CP-MSCs play a vital role in alleviating pancreatic injury and systemic inflammation in SAP rats. These findings provide a safe and effective therapeutic strategy for SAP and also provide new insights into the mechanisms responsible for the effectiveness of exogenous CP-MSCs.

Cell therapy is different from conventional drug therapy, mainly relying on seed cells to secrete a variety of cytokines and active molecules to exert a therapeutic role. Hence, the survival of CP-MSCs was crucial to exert better therapeutic efficacy in SAP. It is imperative to observe the survival status before investigating the therapeutic efficacy of CP-MSCs in SAP rats. The results of bioluminescence imaging revealed that the vast majority of exogenous CP-MSCs survived approximately for $72 \mathrm{~h}$ in the hyperinflammatory environment of SAP, which provided a reference for the selection of CP-MSC treatment endpoint.

Numerous studies have demonstrated that MSCs held the characteristics of migration and colonization to the injury site [32-34]. However, there is no consensus on whether exogenous MSCs colonize the pancreatic injury site in rodent animal models of SAP. Some studies believed that exogenous MSCs mainly resided in the lungs, and almost no MSCs colonized the pancreatic injury site [35]. Nevertheless, other studies suggested that MSCs could colonize the pancreas injury site, and also found that MSCs could differentiate into acinar-like cells $[23,36$, 37]. In this study, we found that CP-MSCs partially colonized the lungs, and we also observed a large number of CP-MSCs in extrapulmonary organs, such as the liver, spleen, and intestine, etc. SAP is often accompanied by obvious intestinal and lung injury, while the heart and kidney generally have no obvious organic injury. We observed that plenty of CP-MSCs were colonized the pancreas, liver, duodenum, and colon of SAP rats, but only few CP-MSCs colonized the heart and kidney with abundant blood flow (Fig. S2). Furthermore, the number of CP-MSCs colonizing in the pancreas, liver, duodenum, and colon of SAP rats was significantly higher than that of control rats. Therefore, it showed that exogenous CP-
MSCs owned the characteristics of colonization at the injured tissue of SAP. The colonization of CP-MSCs at the injury site will be more conducive to exert the therapeutic efficacy in SAP. Although, in rodent animal models of experimental SAP, MSCs tended to migrate and colonize the pancreas injury site, and some studies have confirmed that MSCs colonized in the pancreatic injury site could differentiate into acinar-like cells, the current mainstream view is that MSCs rely on the secretion of various cytokines or active molecules to play a protective role for SAP.

In the present study, one important discovery was that CP-MSCs alleviate pancreatic injury and systemic inflammation by inducing macrophages to polarize from M1 to M2 in SAP rats. Macrophages play a vital role in the progression from local inflammation of the pancreas to a systemic inflammation and multiple organ dysfunction, which makes macrophages an interesting therapeutic target for SAP. In this study, one interesting finding was that macrophages mainly showed M1 phenotype in the pancreas and liver tissues of SAP rats, and exogenous CP-MSCs could induce macrophage polarization from M1 to M2 phenotype. Another interesting finding was that when SAP rats were given simultaneously $\mathrm{GdCl} 3$ and $\mathrm{CP}-\mathrm{MSCs}$, the therapeutic efficacy of CP-MSCs was significantly enhanced, while when SAP rats were given simultaneously MCLs and CPMSCs, the therapeutic efficacy of CP-MSCs was significantly weakened. This result might be explained by the fact that when $\mathrm{GdCl} 3$ and $\mathrm{CP}-\mathrm{MSCs}$ were administered simultaneously in SAP rats, CP-MSCs promoted differentiation of monocytes towards anti-inflammatory macrophages and induced macrophage M2 polarization; meanwhile, GdCL3 depleted M1 macrophages by inducing apoptosis of M1 macrophages, so the proportion of M2 macrophages in the tissue obviously increased; when MCLs and CP-MSCs were administered simultaneously, $\mathrm{CP}-\mathrm{MSCs}$ induced M2 polarization of macrophages; meantime, MCLs depleted M2 macrophages by inducing apoptosis of M2 macrophages, so the proportion of M2 macrophages in the tissues reduced significantly. M1 macrophages are pro-inflammatory macrophages, which secrete a vast array of pro-inflammatory factors (such as IL-1 $\beta$, TNF- $\alpha$, and iNOS) to aggravate the pancreatic and systemic inflammatory response, and M2 macrophages are anti-inflammatory macrophages, which secrete large amounts of anti-inflammatory factors (such as IL-10 and IL-4) to alleviate the pancreatic and systemic inflammatory response $[27,38]$. Therefore, we can infer that CP-MSCs reduce pancreatic injury and systemic inflammatory response by inducing M2 polarization of macrophages in SAP rats.

Another important discovery was that CP-MSCs induced M2 polarization of macrophages by secreting TSG-6, and TSG-6 played a vital role in alleviating 
pancreatic injury and systemic inflammation in SAP rats. Tumor necrosis factor- $\alpha$-induced gene/protein 6 (TSG6) is an inflammation-inducing protein that can reduce tissue inflammation and promote damaged tissue repair in inflammatory diseases. For instance, Choi et al. found that human bone marrow-derived MSCs (BM-MSCs) could attenuate zymosan-induced mouse peritonitis by secreting TSG-6 to inhibit the production of proinflammatory factors of macrophages [39]. Qi et al. findings demonstrated that BM-MSCs accelerated wound healing and reduced tissue fibrosis by secreting TSG- 6 in murine full-thickness skin wounds [40]. Song et al. revealed that BM-MSCs inhibited inflammatory neovascularization in the cornea by suppressing pro-angiogenic monocyte/macrophage recruitment in a TSG-6dependent manner [41]. Meanwhile, some studies have shown that TSG-6 could induce the polarization of macrophages from a pro-inflammatory phenotype (M1) to an anti-inflammatory phenotype (M2). For instance, TSG-6 released from canine adipose tissue-derived (cAT)-MSCs could alleviate dextran sulfate sodiuminduced colitis by inducing a macrophage phenotypic switch to M2 in mice [42]. Moreover, another study showed that TSG-6 secreted by human adipose tissuederived (hAT)-MSCs induced macrophages that infiltrated into the colon to switch to the M2 phenotype, thus regulating the expression of inflammatory cytokines and the alleviation of DSS-induced colitis symptoms in

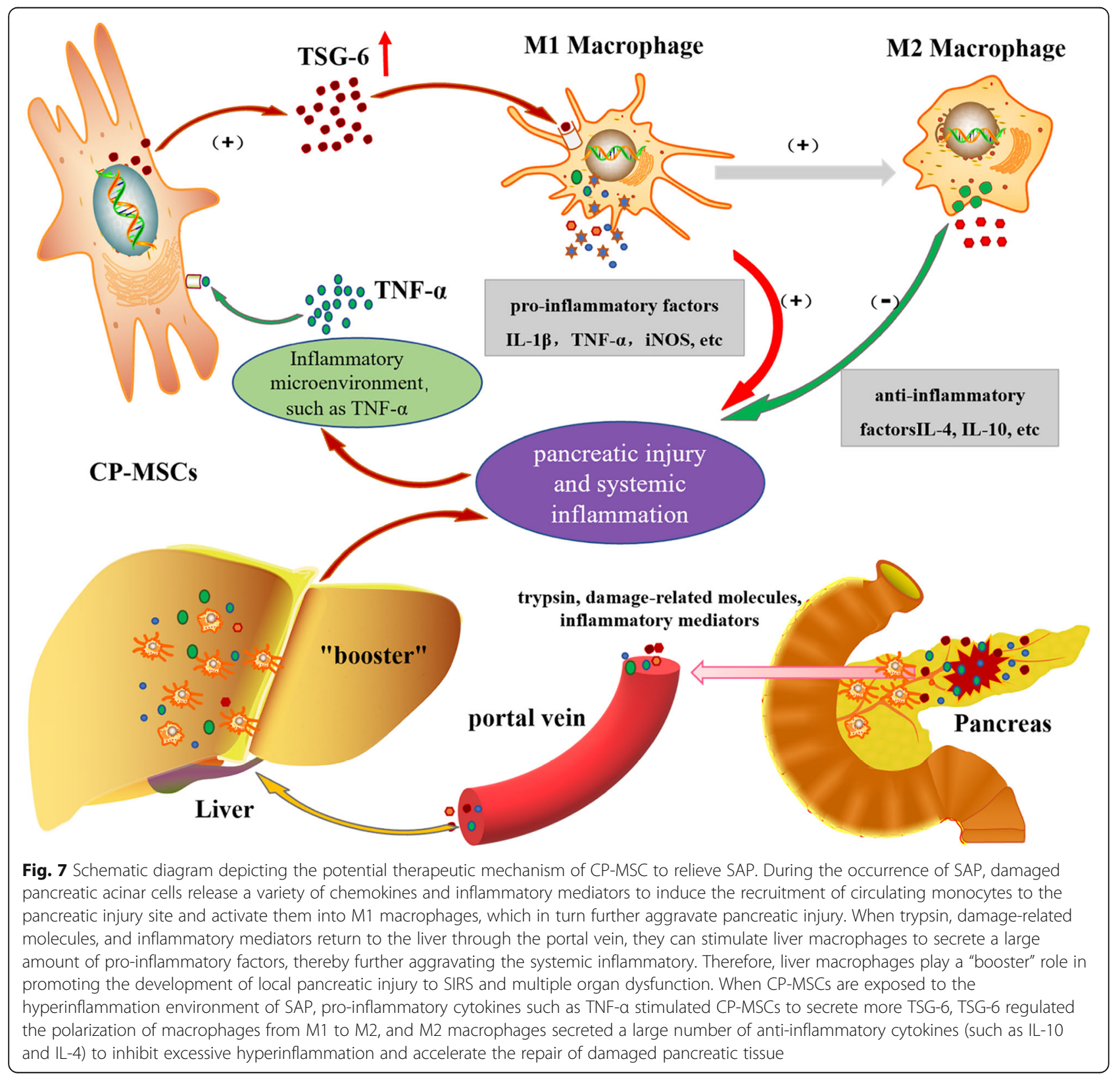


mice [43]. Therefore, we speculated that CP-MSCs might regulate macrophage polarization by secreting TSG-6, thereby reducing pancreatic damage and systemic inflammation in SAP rats.

To confirm this hypothesis, we used RNA interference to knock down the expression of TSG-6 in CPMSCs, and then co-cultured CP-MSCs (TSG-6 shRNA) with M1 macrophages in vitro. Meanwhile, CP-MSCs (TSG-6 shRNA) were transplanted into SAP rats to observe their treatment effects. When suppressing the expression of TSG-6 in CP-MSCs, we found that the ability of $\mathrm{CP}-\mathrm{MSCs}$ to regulate the polarization of macrophages from M1 to M2 was significantly inhibited, and the protective effect of CPMSCs on SAP rats was also significantly weakened. Therefore, it is shown that TSG-6 secreted by CPMSCs exerted an important therapeutic role in SAP rats. Furthermore, we found that the inflammatory environment stimulated CP-MSCs to express TSG-6 higher. Meanwhile, studies have shown that when MSCs are placed in the inflammatory microenvironment, pro-inflammatory cytokines, such as TNF- $\alpha$, stimulate MSCs to secrete TSG-6 [44, 45]. Taken together, we speculated that pro-inflammatory cytokines such as TNF- $\alpha$ stimulated CP-MSCs to secrete more TSG-6 in the inflammatory environment of SAP and TSG-6 regulated the polarization of macrophages from M1 to M2 (Fig. 7).

\section{Conclusion}

In conclusion, our study provides a new treatment strategy for SAP and initially explains the potential protective mechanism of CP-MSCs on SAP rats. We found that CP-MSCs secreted TSG- 6 to induce macrophages to polarize from M1 to M2, thereby reducing pancreatic injury and systemic inflammation in SAP rats. Despite the advancement in our understanding of the therapeutic effects of CP-MSCs in SAP, further study should be taken up using different animal models of SAP.

\begin{abstract}
Abbreviations
ANOVA: Analysis of variance; Arg-1: Arginase-1; BM-MSCs: Bone marrowderived MSCs; CP-MSCs: Chorionic plate-derived MSCs; DMEM: Dulbecco's modified Eagle medium; ELISA: Enzyme-linked immunosorbent assay; FBS: Fetal bovine serum; GdCl3: Gadolinium (III) chloride; IFN: Interferon; IL: Interleukin; iNOS: Inducible nitric oxide synthase; LPS: Lipopolysaccharide; MCLs: Mannosylated clodronate-encapsulated liposomes; M-

CSF: Macrophage colony-stimulating factor; MPO: Myeloperoxidase; MSCs: Mesenchymal stem cells; P-MSCs: Placental-derived MSCs; SAP: Severe acute pancreatitis; SIRS: Systemic inflammatory response syndrome; TCA: Sodium taurocholate; TGF: Transforming growth factor; TNF-a: Tumor necrosis factor-a; TSG-6: Tumor necrosis factor-a-induced gene/protein 6; UC-MSCs: Umbilical cord-derived MSCs
\end{abstract}

\section{Supplementary Information}

The online version contains supplementary material available at https://doi. org/10.1186/s13287-021-02411-9.
Additional file 1: Experimental procedures. Table S1. Primer used for real-time quantitative PCR (RT-qPCR). Figure S1. CP-MSCs isolated from human placental chorionic plate MSCs meet the criteria of MSCs proposed by the ISCT. Figure S2. The distribution of CP-MSCS labeled with CM-Dil in the heart and kidney of rats with or without SAP. Figure $\mathbf{S 3 .}$ Isolation and identification of bone marrow macrophages.

\section{Acknowledgements}

We thank the Pancreatic Injury and Repair Key Laboratory of Sichuan Province for the use of instrumentation and expert assistance. We are also very grateful for the technical guidance of the Department of General Surgery experts at the General Hospital of Western Theater Command.

\section{Authors' contributions}

$\mathrm{HS}$ and $\mathrm{QH}$ conceived the project and designed the experiments. QH and $X C$ performed the majority of the experiments, analyzed the data, and drafted the manuscript. CL and SL participated in some cell experiments. BW, $X Y$, and $Y Y$ are involved in some molecular experiments. YW and RL helped in performing the analysis with constructive discussions. HS and LT revised the final version. All authors read and approved the final manuscript.

\section{Funding}

This work was supported by a grant from the National Natural Science Foundation of China (No. 81772001), National Clinical Key Subject of China (No. 41792113), Technology Plan Program of Sichuan Province (2018JY0041 and 2019YJ0277), and Joint Research Project of the General Hospital of Western Theater (No. 2019LH04).

\section{Availability of data and materials}

All data generated or analyzed during this study are included in this published article.

\section{Declarations}

Ethics approval and consent to participate

This study was approved by the Institutional Animal Care and Use Committee at the General Hospital of Western Theater Command (2019ky179).

\section{Consent for publication}

Not applicable.

\section{Competing interests}

The authors declare that they have no competing interests.

\section{Author details}

'Department of General Surgery \& Pancreatic Injury and Repair Key Laboratory of Sichuan Province, The General Hospital of Western Theater Command, Chengdu 610083, China. ${ }^{2}$ Tianjin Medical University, Tianjin 300070, China. ${ }^{3}$ XinDu Hospital of Traditional Chinese Medicine \& Chengdu 2nd Hospital of Traditional Chinese Medicine, Chengdu 610500, China. ${ }^{4}$ Division of Hepatobiliary Pancreatic Surgery, Panzhihua Central Hospital, Sichuan Province, Panzhihua 617017, China. 'Laboratory of Basic Medicine, The General Hospital of Western Theater Command, Chengdu 610031, China.

Received: 25 January 2021 Accepted: 24 May 2021

Published online: 10 June 2021

\section{References}

1. Zheng L, Xue J, Jaffee EM, Habtezion A. Role of immune cells and immunebased therapies in pancreatitis and pancreatic ductal adenocarcinoma. Gastroenterology. 2013;144(6):1230-40. https://doi.org/10.1053/j.gastro.2 012.12.042.

2. Gukovskaya A, Gukovsky I, Algül H, Habtezion A. Autophagy, inflammation, and immune dysfunction in the pathogenesis of pancreatitis. Gastroenterology. 2017;153(5):1212-26. https://doi.org/10.1053/j.gastro.2017. 08.071.

3. Forsmark CE, Vege SS, Wilcox CM. Acute pancreatitis. N Engl J Med. 2016; 375(20):1972-81. https://doi.org/10.1056/NEJMra1505202. 
4. Shi Y, Wang Y, Li Q, Liu K, Hou J, Shao C, et al. Immunoregulatory mechanisms of mesenchymal stem and stromal cells in inflammatory diseases. Nat Rev Nephrol. 2018;14(8):493-507. https://doi.org/10.1038/s41 581-018-0023-5.

5. Li H, Wang C, He T, Zhao T, Chen YY, Shen YL, et al. Mitochondrial transfer from bone marrow mesenchymal stem cells to motor neurons in spinal cord injury rats via gap junction. Theranostics. 2019;9(7):2017-35. https://doi. org/10.7150/thno.29400.

6. Li C, Jin Y, Wei S, Sun Y, Jiang L, Zhu Q, et al. Hippo signaling controls NLR family pyrin domain containing 3 activation and governs immunoregulation of mesenchymal stem cells in mouse liver injury. Hepatology. 2019;70(5): 1714-31. https://doi.org/10.1002/hep.30700.

7. Walter J, Ware LB, Matthay MA. Mesenchymal stem cells: mechanisms of potential therapeutic benefit in ARDS and sepsis. Lancet Respir Med. 2014; 2(12):1016-26. https://doi.org/10.1016/S2213-2600(14)70217-6.

8. Wu M, Zhang R, Zou Q, Chen Y, Zhou M, Li X, et al. Comparison of the biological characteristics of mesenchymal stem cells derived from the human placenta and umbilical cord. Sci Rep. 2018;8(1):5014. https://doi. org/10.1038/s41598-018-23396-1.

9. Ma J, Wu J, Han L, Jiang X, Yan L, Hao J, et al. Comparative analysis of mesenchymal stem cells derived from amniotic membrane, umbilical cord, and chorionic plate under serum-free condition. Stem Cell Res Ther. 2019; 10(1):19. https://doi.org/10.1186/s13287-018-1104-X.

10. Shen C, Yang C, Xu S, Zhao H. Comparison of osteogenic differentiation capacity in mesenchymal stem cells derived from human amniotic membrane (AM), umbilical cord (UC), chorionic membrane (CM), and decidua (DC). Cell Biosci. 2019;9(1):17. https://doi.org/10.1186/ s13578-019-0281-3.

11. Abedian Z, Jenabian N, Moghadamnia AA, Zabihi E, Pourbagher R, HosseinNataj $\mathrm{H}$, et al. A comparative study on immunophenotypic characterization and osteogenic differentiation of human mesenchymal stromal cells derived from periodontal ligament and gingiva. J Periodontol. 2020;91(9): 1194-202. https://doi.org/10.1002/JPER.19-0535.

12. Talwadekar MD, Kale VP, Limaye LS. Placenta-derived mesenchymal stem cells possess better immunoregulatory properties compared to their cordderived counterparts-a paired sample study. Sci Rep. 2015;5(1):15784. https://doi.org/10.1038/srep15784.

13. Huang $Q$, Yang $Y$, Luo C, Wen Y, Liu R, Li S, et al. An efficient protocol to generate placental chorionic plate-derived mesenchymal stem cells with superior proliferative and immunomodulatory properties. Stem Cell Res Ther. 2019;10(1):301. https://doi.org/10.1186/s13287-019-1405-8.

14. Yang ZX, Han ZB, Ji YR, Wang YW, Liang L, Chi Y, et al. CD106 identifies a subpopulation of mesenchymal stem cells with unique immunomodulatory properties. Plos One. 2013;8(3):e59354. https://doi.org/10.1371/journal.pone. 0059354.

15. Pan LL, Deng YY, Wang R, Wu C, Li J, Niu W, et al. Lactose induces phenotypic and functional changes of neutrophils and macrophages to alleviate acute pancreatitis in mice. Front Immunol. 2018;9:751. https://doi. org/10.3389/fimmu.2018.00751

16. Shrivastava P, Bhatia M. Essential role of monocytes and macrophages in the progression of acute pancreatitis. World J Gastroenterol. 2010;16(32): 3995-4002. https://doi.org/10.3748/wjg.v16.i32.3995.

17. Saeki K, Kanai T, Nakano M, Nakamura Y, Miyata N, Sujino T, et al. CCL2induced migration and SOCS3-mediated activation of macrophages are involved in cerulein-induced pancreatitis in mice. Gastroenterology. 2012; 142(4):1010-1020.e9.

18. Jung M, Ma Y, lyer RP, DeLeon-Pennell KY, Yabluchanskiy A, Garrett MR, et al. IL-10 improves cardiac remodeling after myocardial infarction by stimulating M2 macrophage polarization and fibroblast activation. Basic Res Cardiol. 2017;112(3):33. https://doi.org/10.1007/s00395-017-0622-5.

19. Rahman N, Pervin M, Kuramochi M, Karim MR, Izawa T, Kuwamura M, et al. M1/M2-macrophage polarization-based hepatotoxicity in d-galactosamineinduced acute liver injury in rats. Toxicol Pathol. 2018;46(7):764-76. https:// doi.org/10.1177/0192623318801574.

20. Dayan V, Yannarelli G, Billia F, Filomeno P, Wang XH, Davies JE, et al. Mesenchymal stromal cells mediate a switch to alternatively activated monocytes/macrophages after acute myocardial infarction. Basic Res Cardiol. 2011;106(6):1299-310. https://doi.org/10.1007/s00395-011-0221-9.

21. Morrison TJ, Jackson MV, Cunningham EK, Kissenpfennig A, McAuley DF, O'Kane CM, et al. Mesenchymal stromal cells modulate macrophages in clinically relevant lung injury models by extracellular vesicle mitochondrial transfer. Am J Respir Crit Care Med. 2017;196(10):1275-86. https://doi.org/1 0.1164/rccm.201701-01700C.

22. Liu RH, Wen Y, Sun HY, Liu CY, Zhang YF, Yang Y, et al. Abdominal paracentesis drainage ameliorates severe acute pancreatitis in rats by regulating the polarization of peritoneal macrophages. World J Gastroenterol. 2018;24(45):5131-43. https://doi.org/10.3748/wjg.v24.145.5131.

23. Jung KH, Song SU, Yi T, Jeon MS, Hong SW, Zheng HM, et al. Human bone marrow-derived clonal mesenchymal stem cells inhibit inflammation and reduce acute pancreatitis in rats. Gastroenterology. 2011;140(3):998-1008. https://doi.org/10.1053/j.gastro.2010.11.047.

24. Schmidt J, Rattner DW, Lewandrowski K, Compton CC, Mandavilli U, Knoefel WT, et al. A better model of acute pancreatitis for evaluating therapy. Ann Surg. 1992;215(1):44-56. https://doi.org/10.1097/00000658-1 99201000-00007.

25. Trouplin V, Boucherit N, Gorvel L, Conti F, Mottola G, Ghigo E. Bone marrow-derived macrophage production. J Vis Exp. 2013;81:e50966.

26. Manzanero S. Generation of mouse bone marrow-derived macrophages. Methods Mol Biol. 2012;844:177-81. https://doi.org/10.1007/978-1-61779-52 7-5_12.

27. Murray PJ. Macrophage polarization. Annu Rev Physiol. 2017;79(1):541-66. https://doi.org/10.1146/annurev-physiol-022516-034339.

28. Li J, Xue H, Li T, Chu X, Xin D, Xiong Y, et al. Exosomes derived from mesenchymal stem cells attenuate the progression of atherosclerosis in ApoE(-/-) mice via miR-let7 mediated infiltration and polarization of M2 macrophage. Biochem Biophys Res Commun. 2019;510(4):565-72. https:// doi.org/10.1016/j.bbrc.2019.02.005.

29. Abdel-Zaher AO, Abdel-Rahman MM, Hafez MM, Omran FM. Role of nitric oxide and reduced glutathione in the protective effects of aminoguanidine, gadolinium chloride and oleanolic acid against acetaminophen-induced hepatic and renal damage. Toxicology. 2007;234(1-2):124-34. https://doi. org/10.1016/j.tox.2007.02.014.

30. Serrats J, Schiltz JC, García-Bueno B, van Rooijen N, Reyes TM, Sawchenko $P E$. Dual roles for perivascular macrophages in immune-to-brain signaling. Neuron. 2010;65(1):94-106. https://doi.org/10.1016/j.neuron.2009.11.032.

31. Miron VE, Boyd A, Zhao JW, Yuen TJ, Ruckh JM, Shadrach JL, et al. M2 microglia and macrophages drive oligodendrocyte differentiation during CNS remyelination. Nat Neurosci. 2013;16(9):1211-8. https://doi.org/10.1038/ nn.3469.

32. Karp JM, Leng Teo GS. Mesenchymal stem cell homing: the devil is in the details. Cell stem cell. 2009;4(3):206-16. https://doi.org/10.1016/j.stem.2009. 02.001.

33. Zhao W, Phinney DG, Bonnet D, Dominici M, Krampera M. Mesenchymal stem cell biodistribution, migration, and homing in vivo. Stem Cells Int. 2014;2014:292109.

34. Xiao Q, Wang SK, Tian H, Xin L, Zou ZG, Hu YL, et al. TNF-alpha increases bone marrow mesenchymal stem cell migration to ischemic tissues. Cell Biochem Biophys. 2012;62(3):409-14. https://doi.org/10.1007/s12013-011-9317-y.

35. He Z, Hua J, Qian D, Gong J, Lin S, Xu C, et al. Intravenous hMSCs ameliorate acute pancreatitis in mice via secretion of tumor necrosis factoralpha stimulated gene/protein 6. Sci Rep. 2016;6(1):38438. https://doi.org/1 $0.1038 /$ srep38438.

36. Qu B, Chu Y, Zhu F, Wang B, Liu T, Yu B, et al. Granulocyte colonystimulating factor enhances the therapeutic efficacy of bone marrow mesenchymal stem cell transplantation in rats with experimental acute pancreatitis. Oncotarget. 2017;8(13):21305-14. https://doi.org/10.18632/ oncotarget.15515.

37. Jung KH, Yi T, Son MK, Song SU, Hong SS. Therapeutic effect of human clonal bone marrow-derived mesenchymal stem cells in severe acute pancreatitis. Arch Pharm Res. 2015;38(5):742-51. https://doi.org/10.1007/s122 72-014-0465-7.

38. Martinez FO, Helming L, Gordon S. Alternative activation of macrophages: an immunologic functional perspective. Annu Rev Immunol. 2009;27(1):45183. https://doi.org/10.1146/annurev.immunol.021908.132532.

39. Choi H, Lee RH, Bazhanov N, Oh JY, Prockop DJ. Anti-inflammatory protein TSG-6 secreted by activated MSCs attenuates zymosan-induced mouse peritonitis by decreasing TLR2/NF-kappaB signaling in resident macrophages. Blood. 2011;118(2):330-8. https://doi.org/10.1182/blood-201 0-12-327353.

40. Qi Y, Jiang D, Sindrilaru A, Stegemann A, Schatz S, Treiber N, et al. TSG-6 released from intradermally injected mesenchymal stem cells accelerates wound healing and reduces tissue fibrosis in murine full-thickness skin 
wounds. J Invest Dermatol. 2014;134(2):526-37. https://doi.org/10.1038/jid.2 013.328 .

41. Song HB, Park SY, Ko JH, Park JW, Yoon CH, Kim DH, et al. Mesenchymal stromal cells inhibit inflammatory lymphangiogenesis in the cornea by suppressing macrophage in a TSG-6-dependent manner. Mol Ther. 2018; 26(1):162-72. https://doi.org/10.1016/j.ymthe.2017.09.026.

42. Song WJ, Li Q, Ryu MO, Ahn JO, Bhang DH, Jung YC, et al. TSG-6 released from intraperitoneally injected canine adipose tissue-derived mesenchymal stem cells ameliorate inflammatory bowel disease by inducing $\mathrm{M} 2$ macrophage switch in mice. Stem Cell Res Ther. 2018;9(1):91. https://doi. org/10.1186/s13287-018-0841-1.

43. Song WJ, Li Q, Ryu MO, Ahn JO, Ha Bhang D, Chan Jung Y, et al. TSG-6 secreted by human adipose tissue-derived mesenchymal stem cells ameliorates DSS-induced colitis by inducing M2 macrophage polarization in mice. Sci Rep. 2017;7(1):5187. https://doi.org/10.1038/s41598-017-04766-7.

44. Prockop DJ, Oh JY. Mesenchymal stem/stromal cells (MSCs): role as guardians of inflammation. Mol Ther. 2012;20(1):14-20. https://doi.org/10.1 038/mt.2011.211.

45. He Z, Hua J, Song Z. Concise review: Mesenchymal stem cells ameliorate tissue injury via secretion of tumor necrosis factor-a stimulated protein/ gene 6. Stem Cells Int. 2014;2014:761091.

\section{Publisher's Note}

Springer Nature remains neutral with regard to jurisdictional claims in published maps and institutional affiliations.

Ready to submit your research? Choose BMC and benefit from:

- fast, convenient online submission

- thorough peer review by experienced researchers in your field

- rapid publication on acceptance

- support for research data, including large and complex data types

- gold Open Access which fosters wider collaboration and increased citations

- maximum visibility for your research: over $100 \mathrm{M}$ website views per year

At $\mathrm{BMC}$, research is always in progress.

Learn more biomedcentral.com/submissions 